\title{
Exploring the food environment of young people: Is there a relationship between out of home food outlets and dietary intake?
}

\author{
R. L. Tyrrell ${ }^{1}$, A. A. Lake $^{2}$, S. Hodgson ${ }^{3}$, W. J. Wills ${ }^{4}$ and A. J. Adamson ${ }^{1}$ \\ ${ }^{1}$ Institute of Health and Society, Human Nutrition Research Centre, Newcastle University, Newcastle NE2 4HH, UK, \\ ${ }^{2}$ Centre for Public Policy and Health, School of Medicine and Health, Wolfson Research Institute, Durham University, \\ Stockton-on-Tees TS17 6BH, UK, ${ }^{3}$ MRC-HPA Centre for Environment and Health, Department of Epidemiology and \\ Biostatistics, Imperial College London, London W2 IPG, UK and ${ }^{4}$ Centre for Research in Primary and Community Care, \\ University of Hertfordshire, Hatfield AL10 9AB, UK
}

Obesity is a significant problem in young adults; about one third of 16-24 year olds are overweight or obese ${ }^{(1)}$ and this figure is predicted to rise ${ }^{(2)}$. Relatively little is known about the environmental factors which may influence the food choice and eating habits of young adults $^{(3)}$. Food choices are made within the food environment, which encompasses any opportunity to obtain food or influence food choice $^{(4)}$. Eating outside of the home is associated with higher intakes of energy and fat, and lower micronutrient intakes ${ }^{(5)}$. This study aimed to identify the sources of food most commonly used by young people and investigate the relationship between out of home food outlets and dietary intake.

Participants $(n=45$; mean age $=17$ years; 17 males, 28 females $)$ completed detailed 4-day food diaries. Mean daily intake of food and nutrients was calculated for each participant and the food source (home, friend, school, work, food outlet) was recorded for each eating event. Where the food source was a food outlet, additional information such as the business name and location were recorded. Food outlets were then categorised using a 15-point classification tool ${ }^{(6)}$.

A total of 1040 eating events were recorded by participants (mean $=5.8 /$ person/day, range $=2.75-10.25$ ). The home was the main source of food $(62 \%)$, followed by food outlets $(20 \%)$. Ninety-six per cent of participants used a food outlet at least once over the 4-day period $($ mean $=1.2$ visits/person/day, range $=0-13$ ). Of these, food was most commonly sourced from takeaway and fast food outlets (28 users, 54 eating events), convenience stores (28 users, 38 eating events), supermarkets (18 users, 30 eating events), and restaurants (15 users, 26 eating events). Total number of food outlet eating events was positively associated with mean daily energy density intake $(r=0.329, p=0.03)$. No other significant correlations between food outlet eating events and individual dietary intake were found.

No previous research has linked individual eating behaviour to the food environment with respect to where food is obtained, or the number and type of food outlets which an individual visits. The home is the primary source of food for young adults; however, food for a fifth of eating events is obtained from out of home food outlets. Identifying the types of outlets young people use is required in order to target interventions encouraging healthier food environments and choices.

1. The NHS Information Centre (2011) Health Survey for England 2010: Respiratory Health.

2. Foresight (2007) Scoping the Foresight Project on Tackling Obesities: Future Choices.

3. Contento IR, Williams SS, Michela JL et al. (2006) J Adolesc Health 38 575-582.

4. Townshend T \& Lake AA (2009) Health Place 15, 909-916.

5. Lachat C, Nago E, Verstraeten R et al. (2012) Obes Rev 13, 329-346.

6. Lake AA, Burgoine T, Greenhalgh F et al. (2010) Health Place 16, 666-673. 